\title{
Extremely weak and super-efficient production of keV sterile neutrino: phase transition and induced parametric resonance creation
}

\section{A. Chudaykin*}

Institute for Nuclear Research of the Russian Academy of Sciences, Moscow 117312, Russia Moscow Institute of Physics and Technology, Dolgoprudny 141700, Russia

E-mail: chudy@ms2.inr.ac.ru

\begin{abstract}
We introduce a novel generation mechanism of sterile neutrinos in the presence of a light coherently oscillating scalar field. This field modulates the mass of sterile neutrino in the early Universe providing non-trivial oscillation dynamics. In a region of model parameters oscillations between active and sterile neutrinos are resonantly enhanced. This mechanism allows sterile neutrinos with extremely small mixing to produce Dark Matter (DM) evading X-ray constraints. At the same time the spectrum of produced particles is cooler than in case of ordinary non-resonant production of sterile neutrinos, thus opening a window of lower masses which is otherwise forbidden by structure formation constraints.

This work was made in collaboration with Fedor Bezrukov and Dmitry Gorbunov.
\end{abstract}

The 39th International Conference on High Energy Physics (ICHEP2018)

4-11 July, 2018

Seoul, Korea

${ }^{*}$ Speaker. 


\section{Introduction}

The nature of Dark Matter (DM) remains elusive so far. Sterile neutrino is a well-motivated DM candidate. Such a particle appears in a minimal extension $v$ MSM [1,2] of the Standard Model of particle physics (SM). If one sterile neutrino is light enough with mass $\mathscr{O}(1-100) \mathrm{keV}$ it is stable on cosmological time scales and hence can compose DM today [3]. This scenario is parametrised by sterile neutrino Majorana bare mass $M_{0}$ and mixing angle with the active neutrino in vacuum $\theta_{0}$. The most relevant constraint arrives from two body decay $N \rightarrow v \gamma$ leading to strong X-ray signal from DM dominated regions in the sky which imposes the upper bound on $\theta_{0}$ [4]. At the same time producing the sufficient number of sterile neutrinos $\Omega_{N}=\Omega_{D M}$ requires sizable mixing $\theta_{0}$ according to conventional non-resonant production. The last constraint originates from the investigation of cosmic structure formation which limits the velocity distribution of DM particles. Such tests impose a significant constraint from below on its mass $M$. Thus, production of cold or mildly warm DM particles is required.

In this letter we introduce a novel mechanism of sterile neutrino production operating in the presence of a coherently oscillating scalar field in the early Universe. Oscillation dynamic in such a system is non-trivial. The periodic crossing of the sterile neutrino Majorana mass $M(t)=0 \mathrm{can}$ lead to resonant effects in the active-sterile oscillations. This mechanism provides a cool spectrum and allows us to produce all DM with extremely small mixing.

\section{Induced parametric resonance}

We study oscillations in two level system in the presence of the light scalar field $\phi$

$$
\mathscr{L}=i \bar{v} \not \partial v+i \bar{N} \not \partial N+\frac{1}{2}\left(\partial_{\mu} \phi\right)^{2}+\frac{m_{\phi}^{2}}{2} \phi^{2}+\left(m_{D} \bar{v} N+\frac{M_{0}}{2} \bar{N}^{c} N+\frac{f}{2} \phi \bar{N}^{c} N+\text { h.c. }\right),
$$

where $v$ is an active SM neutrino and $N$ is the right-handed sterile neutrino. Henceforth we always assume $M_{0} \gg m_{D}$. At the present the scalar field is in its symmetric phase $\phi=0$, so diagonalisation of the mass term in (2.1) leads to the present active-sterile mixing

$$
\theta_{0} \simeq m_{D} / M_{0}
$$

The scalar field coherently oscillates in the early Universe, so its amplitude decreases with plasma temperature as $\propto T^{3 / 2}$. This induces the temperature dependent contribution to Majorana mass

$$
M(t)=M_{0}+M_{A} \sin m_{\phi} t, \quad M_{A} \equiv M_{0}\left(\frac{g_{*} T^{3}}{g_{*, e} T_{e}^{3}}\right)^{1 / 2},
$$

where $T_{e}$ is the temperature when $M_{A}=M_{0}$. We note that the frequency of scalar field oscillations $m_{\phi}$ is much bigger than a Hubble rate $H$.

The coherent evolution of neutrino state of given momentum $p$ (dimensionless conformal quantity $y \equiv p / T$ is also used) is driven (in case of relativistic momentum $p \simeq 3 T \gg M(t)$ ) by

$$
i \frac{\partial}{\partial t} \rho=[\mathscr{H}, \rho]-\frac{i}{2}\left\{\Gamma, \rho-\rho_{\mathrm{eq}}\right\} .
$$



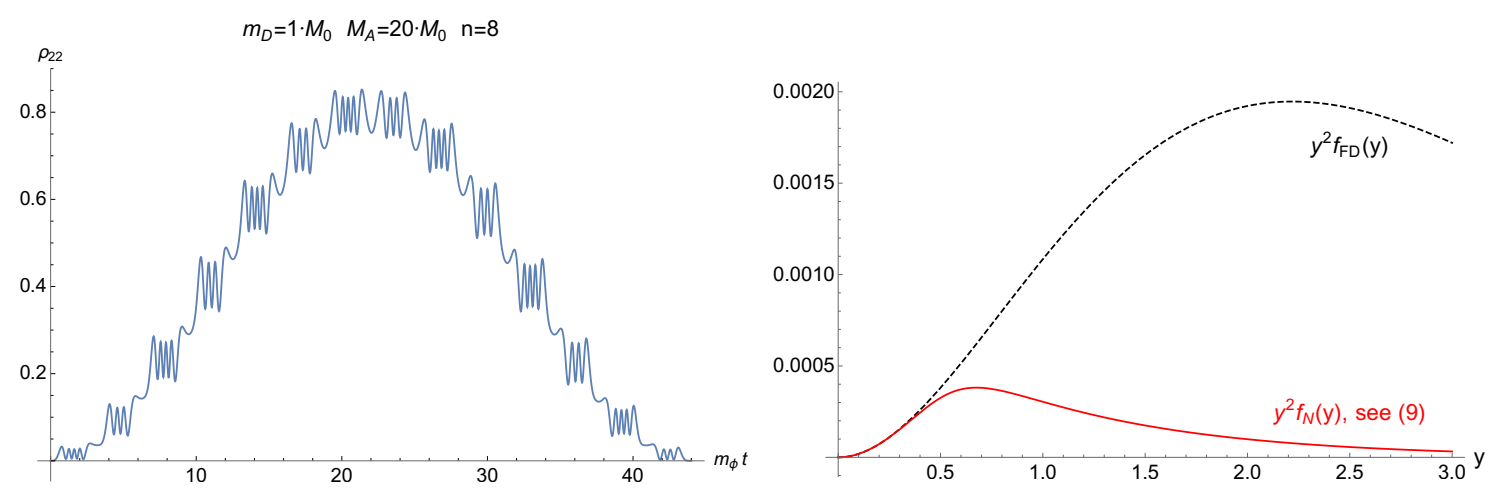

Figure 1: Left: Resonant behaviour of transition probability for sterile state with $n=8$. Relation between $M_{0}$ and $p$ is defined by (2.5). Fast oscillation with rate $M_{A}^{2} / 4 p \approx 8 m_{\phi}$, slower oscillations of frequency $m_{\phi}$ related to scalar field evolution and modulation by resonant frequency (2.6) are shown here. Right: Fermi-Dirac $f_{\mathrm{FD}}$ and sterile neutrino $f_{N}$ spectra.

where $2 \times 2$ density matrix $\rho$ describes the dynamics of active and sterile neutrino states with probability densities $\rho_{11}$ and $\rho_{22}$, respectively. Here $\rho_{\mathrm{eq}}=\operatorname{diag}\left(f_{\mathrm{FD}}(y), f_{\mathrm{FD}}(y)\right)$ is equilibrium FermiDirac distribution and $\Gamma=\left(\begin{array}{cc}\Gamma_{A} & 0 \\ 0 & 0\end{array}\right)$ is a damping term due to neutrino scattering in plasma. The Hamiltonian of such a system reads

$$
\mathscr{H}=\frac{\Delta_{0}}{2}\left(\begin{array}{cc}
-\cos 2 \theta & \sin 2 \theta \\
\sin 2 \theta & \cos 2 \theta
\end{array}\right), \text { where } \Delta_{0}=\frac{\Delta m^{2}}{2 p}
$$

with time-dependent oscillating parameters

$$
\Delta m^{2}=M \sqrt{M^{2}+4 m_{D}^{2}}, \tan \theta=2 m_{D} /\left(M+\sqrt{M^{2}+4 m_{D}^{2}}\right) .
$$

Evolution (2.4) for a given momentum $p_{\text {res }}$ provides resonant behaviour if typical frequency of neutrino oscillation (properly averaged) is an integer multiplier of the frequency of the scalar field oscillations $m_{\phi}$,

$$
\frac{M_{A}^{2}+2 M_{0}^{2}}{4 p_{\text {res }}}=n m_{\phi}, \quad n \in \mathbb{Z} .
$$

It can be shown [5] that in resonance (2.5) without scatterings, $\Gamma_{A}=0$, the oscillations between $v$ and $N$ proceed with maximum probability $\rho_{22} \simeq \sin ^{2}\left(\omega_{\text {res }} t / 2\right)$ at frequency

$$
\omega_{\mathrm{res}} \simeq 1.4 m_{\phi} \frac{m_{D}}{M_{A}} n^{1 / 3} \cos \frac{4 M_{0}}{M_{A}} n \simeq 1.4 m_{\phi} \frac{m_{D}}{M_{A}} n^{1 / 3},
$$

which is a good estimate for $M_{A} \gg M_{0}$. Numerically it works well until $M_{A} \gtrsim 5 M_{0}$.

Note that the chosen parameters resonant frequency (2.6) is much lower than other typical scales of the theory, $m_{\phi}$ and $M_{A}^{2} / 4 p$. The width of this resonance appears to be narrow

$$
\frac{\Delta p_{\mathrm{res}}}{p_{\mathrm{res}}} \simeq \frac{\sqrt{2} \omega_{\mathrm{res}}}{n m_{\phi}} \simeq \frac{2}{n} \frac{m_{D}}{M_{A}} \ll 1
$$


For resonant frequency (2.6) sterile neutrino state reaches equilibrium (Fermi-Dirac) distribution after typic time $\omega_{\text {res }}^{-1}$. However, expansion of the Universe leads to deviations of $p_{\text {res }}$ and $M_{A}$ from their resonant values defined by (2.5) which eventually results in termination of resonance evolution. Namely, if resonant band $\Delta y_{\text {res }}=\Delta p_{\text {res }} / T$ moves through a given $y$ faster than $\omega_{\text {res }}^{-1}$, the resonance becomes too narrow and ineffective. This happens at high temperatures when

$$
\frac{1}{n} \frac{\Delta y_{\text {res }}}{y_{\text {res }}} \frac{y_{\text {res }}}{\dot{y}_{\text {res }}} \omega_{\text {res }}=\frac{1}{n} \frac{\omega_{\text {res }}^{2}}{\sqrt{2} m_{\phi} H} \lesssim 1
$$

We address redshift effects in (2.4) properly and obtain the approximate sterile neutrino spec$\operatorname{trum} f_{N}[5]$

$$
f_{N}(y)=\frac{f_{\mathrm{FD}}(y)}{\sqrt{1+0.12\left(\frac{y}{y_{s}}\right)^{5}}} .
$$

where $y_{s}$ defines a sterile neutrino DM abundance today $\Omega_{D M} \rho_{\text {crit }}=M_{0} 2 \frac{4}{11} T_{0}^{3} \int 4 \pi y^{2} f_{N}(y) d y$

$$
y_{s} \simeq 0.4\left(1 \mathrm{keV} / M_{0}\right)^{2 / 5} .
$$

This spectrum is suppressed at high momenta as shown in Fig. 1.

The correct DM is achieved if the resonance becomes too narrow (2.8) while mode (2.10) is passing through the resonance, leading to the final result for active-sterile mixing at present

$$
\theta_{0} \sim 1.6 \times 10^{-6}\left(\frac{1 \mathrm{keV}}{M_{0}}\right)^{3}\left(\frac{g_{*, s}}{10.75}\right)^{1 / 4}\left(\frac{m_{\phi}}{1 \mathrm{eV}}\right)^{3 / 4} \times\left(\frac{T_{e}}{5.6 \mathrm{MeV}}\right)^{9 / 4} .
$$

The sterile neutrino DM of spectrum (2.9) is cool with average momentum $\langle\langle y\rangle\rangle=1.3$. We apply Ly- $\alpha$ constraint $m_{\mathrm{NRP}}>8 \mathrm{keV}$ [4] leading to

$$
M_{0}>3.3 \mathrm{keV} \text {. }
$$

\section{Conclusion}

We suggest a new generation mechanism of sterile neutrino DM in the early Universe. Oscillations between active and sterile neutrinos can be resonantly-enhanced by oscillating background. In resonant scenario conversions are effective even with small mixing. At the same time, produced this way DM is cool which opens a window for lower masses consistent with structure formation.

The study of resonant production is supported by RSF grant 17-12-01547.

\section{References}

[1] T. Asaka, S. Blanchet and M. Shaposhnikov, Phys. Lett. B 631, 151 (2005) [hep-ph/0503065].

[2] T. Asaka and M. Shaposhnikov, Phys. Lett. B 620, 17 (2005) [hep-ph/0505013].

[3] A. D. Dolgov and S. H. Hansen, Astropart. Phys. 16, 339 (2002) [hep-ph/0009083].

[4] M. Drewes et al., JCAP 1701, no. 01, 025 (2017) [arXiv:1602.04816 [hep-ph]].

[5] Bezrukov, Fedor and Chudaykin, A. and Gorbunov, Dmitry, Scalar Induced Resonance, in preparation, 2018

[6] F. Bezrukov, A. Chudaykin and D. Gorbunov, JCAP 1706, no. 06, 051 (2017) [arXiv:1705.02184 [hep-ph]]. 